\title{
An enhanced fall detection system for elderly person monitoring using consumer home networks
}

Article

Accepted Version

Wang, J., Zhang, Z., Bin, L., Lee, S. and Sherratt, S. (2014) An enhanced fall detection system for elderly person monitoring using consumer home networks. IEEE Transactions on Consumer Electronics, 60 (1). pp. 20-29. ISSN 0098-3063 doi: https://doi.org/10.1109/TCE.2014.6780921 Available at https://centaur.reading.ac.uk/36557/

It is advisable to refer to the publisher's version if you intend to cite from the work. See Guidance on citing.

To link to this article DOI: http://dx.doi.org/10.1109/TCE.2014.6780921

Publisher: IEEE Consumer Electronics Society

All outputs in CentAUR are protected by Intellectual Property Rights law, including copyright law. Copyright and IPR is retained by the creators or other copyright holders. Terms and conditions for use of this material are defined in the End User Agreement.

www.reading.ac.uk/centaur 
Central Archive at the University of Reading

Reading's research outputs online 


\title{
Full Text of article
}

\section{Title: $\quad$ An Enhanced Fall Detection System for Elderly Person Monitoring using Consumer Home Networks}

\author{
Publication: IEEE Transactions on Consumer Electronics \\ Volume: $\quad \mathbf{6 0}$ \\ Issue: $\quad \mathbf{1}$ \\ pp.: 23-29 \\ URL: $\quad$ http://dx.doi.org/10.1109/TCE.2014.6780921 \\ DOI: $\quad$ 10.1109/TCE.2014.6780921
}

Authors:

Jin Wang, Member, IEEE, School of Computer and Software, Jiangsu Engineering Center of Network Monitoring, Nanjing University of Information Science \& Technology, 210044, China (e-mail: wangjin@ @uist.edu.cn).

Zhongqi Zhang, School of Computer and Software, Jiangsu Engineering Center of Network Monitoring, Nanjing University of Information Science \& Technology, 210044, China (e-mail: zqzhang@nuist.edu.cn).

Bin Li, College of Information Engineering, Yangzhou University, Yangzhou 225009, China (e-mail: lb@yzu.edu.cn).

Sungyoung Lee, Computer Engineering Department, Kyung Hee University, Suwon 449-701, Korea (e-mail: sylee@khu.ac.kr).

R. Simon Sherratt, Fellow, IEEE, School of Systems Engineering, the University of Reading, RG6 6AY, UK (e-mail: sherratt@ieee.org).

This work was supported by the National Natural Science Foundation of China $(61173072,61070133,61271240)$ and the Natural Science Foundation of Jiangsu Province (BK2012461). It was also supported by the Industrial Strategic Technology Development Program (10041740) by the MOTIE Korea, PAPD of Jiangsu Higher Education Institutions, a project funded by Nanjing University of Information Science and Technology (S8110246001) and the National Research Foundation of Korea grant funded by the Korea government (MEST) (2011-0030823).

\begin{abstract}
Various fall-detection solutions have been previously proposed to create a reliable surveillance system for elderly people with high requirements on accuracy, sensitivity and specificity. In this paper, an enhanced fall detection system is proposed for elderly person monitoring that is based on smart sensors worn on the body and operating through consumer home networks. With treble thresholds, accidental falls can be detected in the home healthcare environment. By utilizing information gathered from an accelerometer, cardiotachometer and smart sensors, the impacts of falls can be logged and distinguished from normal daily activities. The proposed system has been deployed in a prototype system as detailed in this paper. From a test group of 30 healthy participants, it was found that the proposed fall detection system can achieve a high detection accuracy of $97.5 \%$, while the sensitivity and specificity are $96.8 \%$ and $98.1 \%$ respectively. Therefore, this system can reliably be developed and deployed into a consumer product for use as an elderly person monitoring device with high accuracy and a low false positive rate.
\end{abstract}

\section{Index Terms}


Wireless Sensor Networks, Fall Detection System, Elderly Monitoring, Heart Rate Fluctuation, Sensitivity. 


\section{Introduction}

In recent years, many types of consumer electronics devices have been developed for home network applications. A consumer home network usually contains various types of electronic devices, e.g. sensors and actuators, so that home users can control them in an intelligent and automatic way to improve their quality of life [1].

Some representative technologies to implement a home network include: IEEE 802.11, Ultra Wide Band (UWB), Bluetooth and ZigBee, etc. ZigBee is suitable for consumer home networks because various sensors can be deployed to collect home data information in a distributed, self-organizing manner with relatively low power. Some typical applications include home automation, home activity detection (like fall detection) and home healthcare, etc. [2].

Kinsella and Phillips [3] found that the population of 65-and-over aged people in the developed countries will approach $20 \%$ of total population in the next 20 years and will obviously become a serious healthcare issue in the near future. In China alone, the population over the age of 60 years old is 133.9 Million [4], [5]. Among the elderly, the fall events can be an unpredictable and dangerous event. Statistics show that one among three 65-and-over aged person falls every year [6]. Among these fall events, $55 \%$ occur at home and $23 \%$ occur near the home. In 2003, the global number of deaths caused by fall events was approximately 391,000 and specifically $40 \%$ of the falls were from people over 70 years of age [7]. Thus, reliable consumer based fall detection systems need to be designed, tested and commercially deployed to countries all around the world. Furthermore, the cost of healthcare is highly related to the response and rescue time, and can be greatly reduced by fast detection and delivering signals to the specified operator for immediate consideration [8]. Thanks to the development of wireless sensors and low-power sensor nodes, many novel approaches have been proposed to solve the problem, as discussed in Section II.

In this paper, an enhanced fall detection system for elderly person monitoring through a consumer home network environment is proposed that based on smart sensors which are worn on the body. The proposed system has been deployed in a prototype system and tested with a group of 30 healthy participants, it is found that the system can achieve very high accuracy of $97.5 \%$, the sensitivity and specificity are $96.8 \%$ and $98.1 \%$ respectively.

The rest of the paper is organized as follows: Section II details related works. Section III describes the system architecture and sensor deployment. Section IV explains the fall detection system in detail. Section V illustrates system performance and Section VI concludes this paper. 


\section{Related Works}

Many previous and current research projects use medical sensor networks to identify and track human activities in daily life. With the purpose to successfully detect falls, there are primarily three types of fall detection methods for elderly people, namely wearable device based methods, vision based methods, and ambient based methods.

\section{A. Wearable Based Methods}

Wearable based methods often rely on smart sensors with embedded processing. They can be attached to the human body or worn in their garments, clothing or jewelry.

Zhang, Ren and Shi [9] proposed HONEY (Home healthcare sentinel system), a three-step detection scheme which consisted of an accelerometer, audio, image and video clips. Its innovation was to detect falls by leveraging a tri-axial accelerometer, speech recognition, and on-demand video. In HONEY, once the fall event was detected, an alert email was immediately sent and the fall video was uploaded to the network storage for further investigation.

Bagalà et al. [10] gave an evaluation of accelerometer-based fall detection algorithms on real-world falls . They found that the sensitivity and specificity on real falls are much lower than that in an experiment environment. This inspires researchers to take more real world scenarios into consideration.

Abbate et al. [11], [12] proposed a smartphone based fall detection system with consideration of the acceleration signal produced by fall-like activities of daily lives.

Bai, $\mathrm{Wu}$ and Tsai [13] illustrated a system based on a 3-axis accelerometer embedded in a smart phone which had a GPS function for the user. However, due to the relatively high energy consumption of current smart phones, their system could only be active for 40 hours with foreground execution or at most 44 hours in background execution, which means continuation of this system is the most significant problem.

\section{B. Vision Based Methods}

Vision based methods are always related to spatiotemporal features, change of shape, and posture.

Yu et al. [14] proposed a vision based fall detection method by applying background subtraction to extract the foreground human body and post processing to improve the result. To detect a fall, information was fed into a directed acyclic graph support vector machine for posture recognition. This system reported a high fall detection rate and low false detection rate.

Rougier et al. [15] analyzed human shape deformation during a video sequence which is used to track the person's silhouette. 


\section{Ambient Based Methods}

Ambient based methods usually rely on pressure sensors, acoustic sensors or even passive infrared motion sensors, which are usually implemented around caretakers' houses [16]-[18].

Popescu et al. [16] developed an acoustic-based fall detection system which used an array of acoustic sensors. The fall detection sensors are linear arrays of electret condensers placed on a pre-amplifier board. In order to capture the information of the sound height, the sensor array was placed in the z-axis. The limitation of this method was that that only one person was allowed in the vicinity.

Winkley, Jiang and Jiang [17] proposed Verity, a 2-component system which had a based station and a direct monitoring device. In this particular system, ambient/skin temperatures were measured for real time monitoring. Experiments verified that the proposed classifier outperforms the conventional classifiers in its one-pass training and with higher distinguishing capability.

Yan et al. [18] addressed the perceived invasive nature of these wearable devices by developing a system that did not necessarily require the user to be wearing a sensor, yet was able to detect the user's location based on observations of interaction with the home-installed sensor network.

Video based methods are usually more accurate than wearable based and ambient based methods. However, these systems often suffer from high risk of privacy and the prohibitive cost implementing the cameras. Thus, wearable sensor based methods are considered in this research.

\section{System Implementation}

The structure of proposed fall detection system is shown in Fig. 1, whose core structure is based on a Microprogrammed Controller Unit (MCU). The accelerometer sensor is complemented by other smart sensors including temperature and humidity sensors all integrated on one single board, recording real time acceleration and ambient environment information. Both acceleration and environment information are first captured using an analog-to-digital converter (ADC). Then, the digital signal is transmitted to the MCU for further processing. The heart rate is captured by a pulse pressure sensor and also passed directly to the MCU. The system is complemented with a customer interface designed to monitor information in real-time.

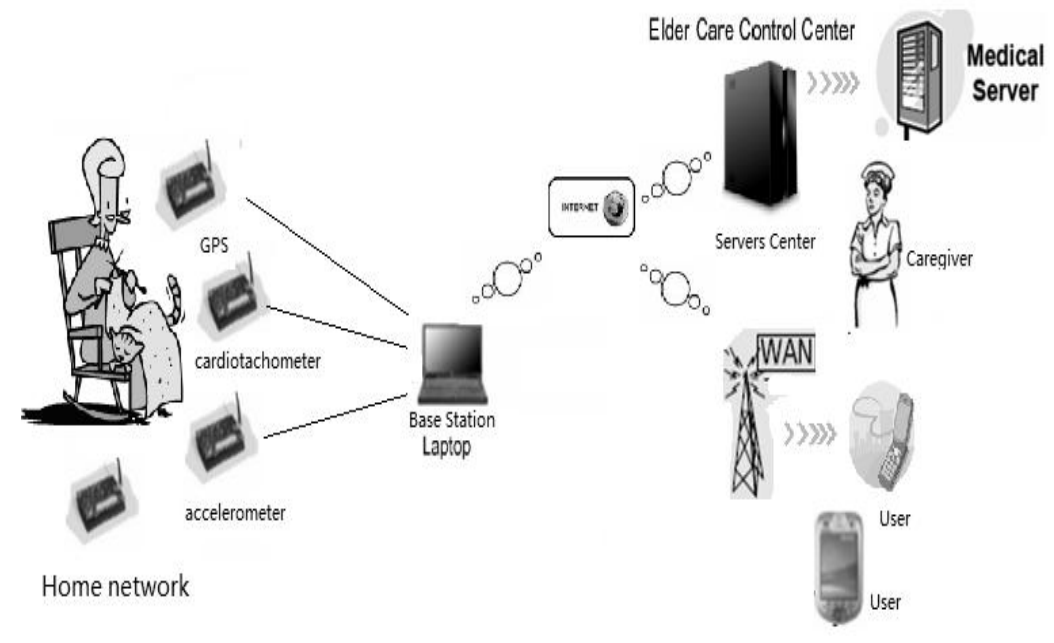

Fig. 1. System architecture using the consumer wireless sensor network. 


\section{A. System and Sensors}

A multi-functional data acquisition board has been used incorporating temperature and humidity sensors. Besides, it offered a convenient solution to add any custom sensing application in future research. To detect the impact of accidental falls, a small low power tri-axial accelerometer is used as shown in Fig. 2. It can measure the static acceleration of gravity in tilt-sensing applications. Also, it can measure the dynamic acceleration results from motion, shock, or vibration. This specified accelerometer will output acceleration in all three axis at every sample point, with units of $\mathrm{m} / \mathrm{s}^{2}$. The output is an analog signal which must be converted by an ADC before sending to the MCU. However, the other smart sensors used in this system are utilized to detect the heartbeat pulse with sensitivity $0.2 \mathrm{mv} / \mathrm{pa}$.

\section{B. MCU System}

The key component of this system is a MCU with $128 \mathrm{~K}$ flash memory. It is a compromise between relatively high performance vs. low-power $(2.7-5.5 \mathrm{~V})$. This high-density nonvolatile memory based MCU provides an embedded 8-channel, 10-bit ADC, and provides a highly flexible and cost effective solution to many embedded control applications. Information gathered by accelerometer is converted in the chip and forwarded to the wireless communication module along with pulse signals.

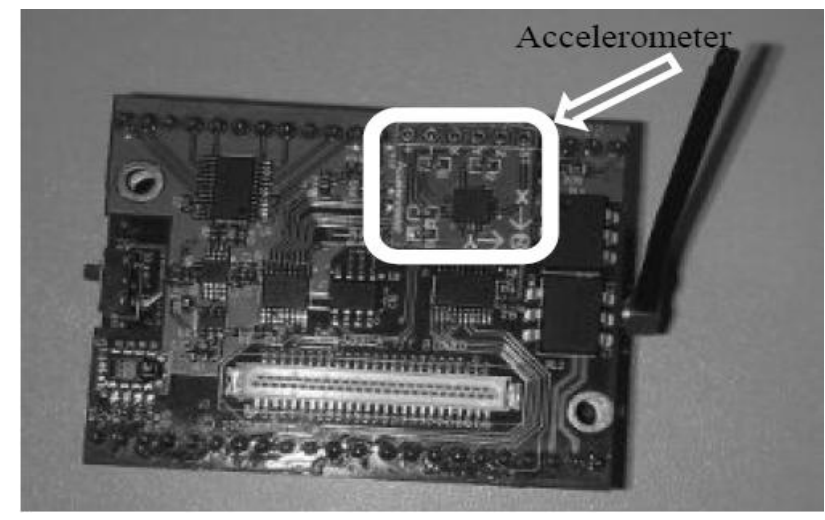

Fig. 2. Wireless communication module with board accelerometer.

\section{User Interface}

The data gathered from a participants body is appended with a unique ID and transmitted to a remote laptop by the wireless receiver with type number of the base station being used. As is shown in Fig. 3, a user interface is designed to display the accelerometer and heart rate signal. The interface can monitor four participants' data at the same time. In each part, data curves are illustrated on upper left and realtime data are shown on the right of the curves. Once the alarm is triggered, a red marked warning will be shown at the bottom left part of the monitors.

In order to assure that a caregiver, or relatives, get real-time and accuracy information, the location of the wireless sensor network is significant. Modern wireless sensor networks have been highly normalized by ZigBee, but they cannot efficiently handle the specific tasks due to the constrained environment. In order to do so, the wireless communication stack in the wireless sensor network needs to be optimized so many sensor nodes need to be put in one base station. Every sensor node can be freely configured as a master or slave. Considering ZigBee transmission power, propagation does not reliably pass through modern construction walls to the base station, therefore the base station usually does not receive the signal transmitted from a neighboring room, as shown in Fig. 4. 
To detect the acceleration and heart rate more accuracy, the whole house can be divided into several clusters based on the room locations. Each room has a fixed access point for data collection and transmission. The sensor nodes represent the accelerometer or cardiotachometer, which could be located anywhere in the house. The signal from wireless module can be transmitted directly to base station or through the fixed access point. The system employs mesh networking to enable communication when it encounters problems of connecting to the base station directly. Fig. 5 indicates how the sensors have been deployed in the patient's home.

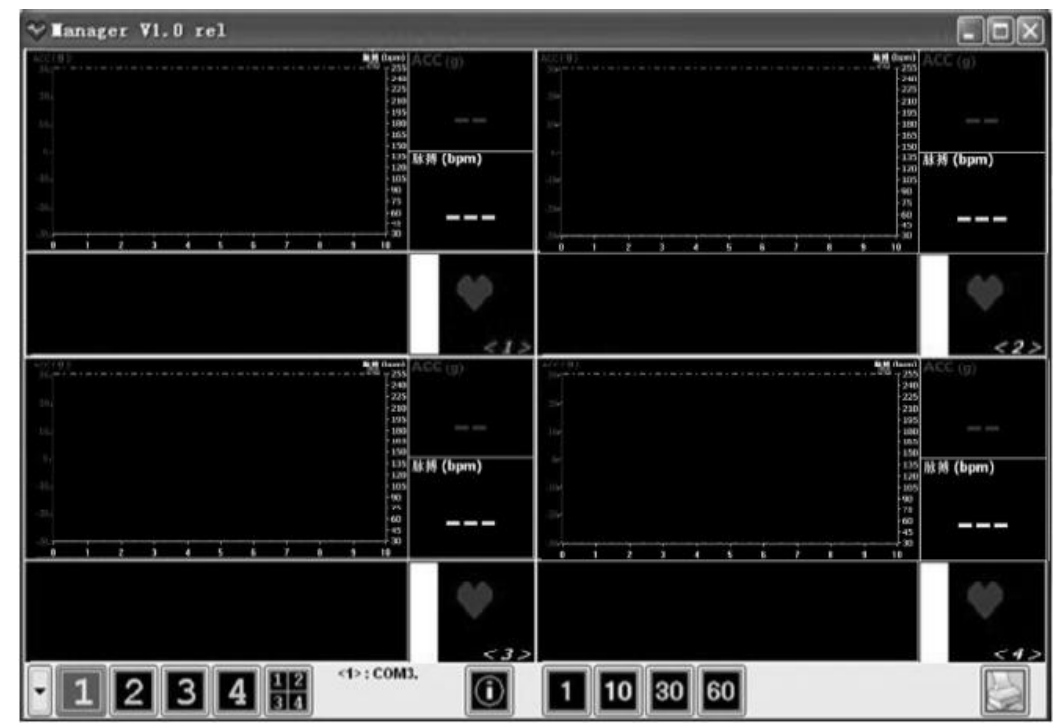

Fig. 3. User interface for the management software

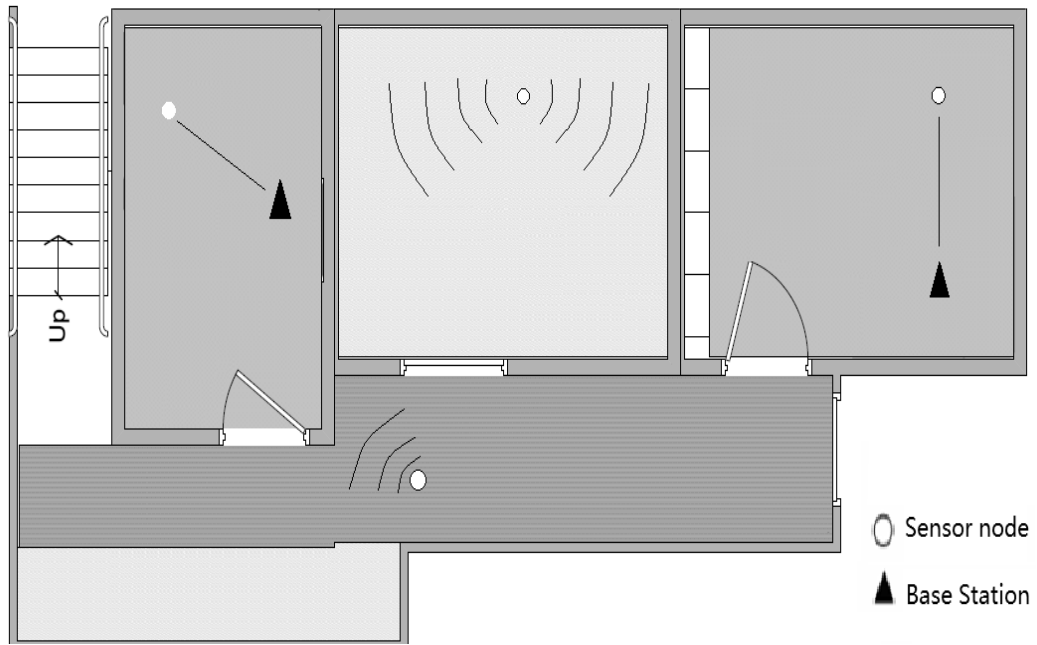

Fig. 4. Sensor and base station deployment 


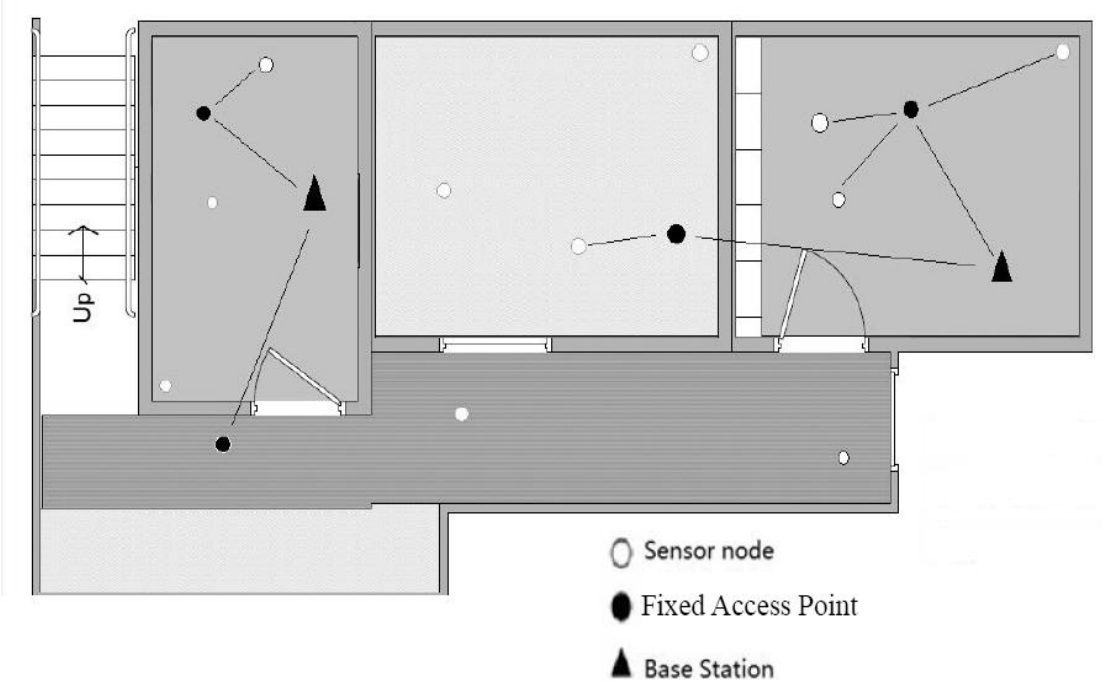

Fig. 5. Sensor, fixed access point and base station deployment

\section{Proposed Enhanced Fall Detection Method}

The proposed enhanced fall detection method is based on three common changes which happen during accidental falls: impact magnitude, trunk angle, and after-event heart rate. Hence, a triple-threshold for the previously fall related event in chronological order is proposed in this paper. A flowchart of the proposed method is illustrated in Fig. 6.

An initiatory estimation of the body movement can be obtained from the Signal Magnitude Vector (SMV) defined as:

$$
S M V=\sqrt{A c c_{x}^{2}+A c c_{y}^{2}+A c c_{z}^{2}}
$$

where $A c c_{x}, A c c_{y}$ and $A c c_{z}$ represent the outputs of $\mathrm{x}$-axial, y-axial and z-axial, respectively. Since the direction of possible falls cannot be predicted, it is inappropriate to use only one output of the axis. The advantage of using equ. (1) is that it is sensitive to all directions of falls. At the beginning, acceleration due to gravity, $g$, lies in the $\mathrm{z}$ direction. The acceleration changes along with body movement, Furthermore, vibration becomes significant when the fall happens. Acceleration threshold had been set to $1.9 \mathrm{~g}$ as in the literature [9].

A typical fall event ends with the person lying on the ground or leaning on walls, or furniture that will cause a significant change in truck angle. In this case, it is desirable to consider changes on the truck angle to detect whether the detected acceleration was due to a fall event. Trunk angle, $\theta$, can be defined as angle between the SMV and positive z-axis and can be calculated by inverse trigonometric function as equ. (2). The threshold for $\theta$ has previously been given as: 0 to $60^{\circ}$ classified as upright and 60 to $120^{\circ}$ classified as a lying posture [19].

$$
\theta=\arccos \left(\frac{A c c_{z}}{S M V}\right)
$$


The emergency case can then be classified into four levels:

1. Caregiver level: When the system is setup, it will check whether the SMV is over threshold. If not, it would continually check the heart rate. Once the heart rate gets over a preset value, the system will assume an emergency event has happened and would contact the caregivers to check out the elderly's condition.

2. Relatives level: Once the system convinced the acceleration is over threshold in the first decide loop, the system will then examine the value of heart rate. If it does not get higher than the preset threshold, then relatives will be contacted to request the relatives contact the elderly person's home.

3. Caregiver and relatives level: In addition, in case the acceleration and heart rate value both get higher than the preset thresholds, then system can contact the caregivers and relatives irrespective of the trunk angle as a distinct floating in heart rate coupled with high acceleration is a significant warning.

4. Ambulance level: If all three thresholds, SMV, heart rate, and trunk angle, are higher than normal, the system as assumed that an accidental fall has happened. The system will contact the emergency center immediately requiring an ambulance.

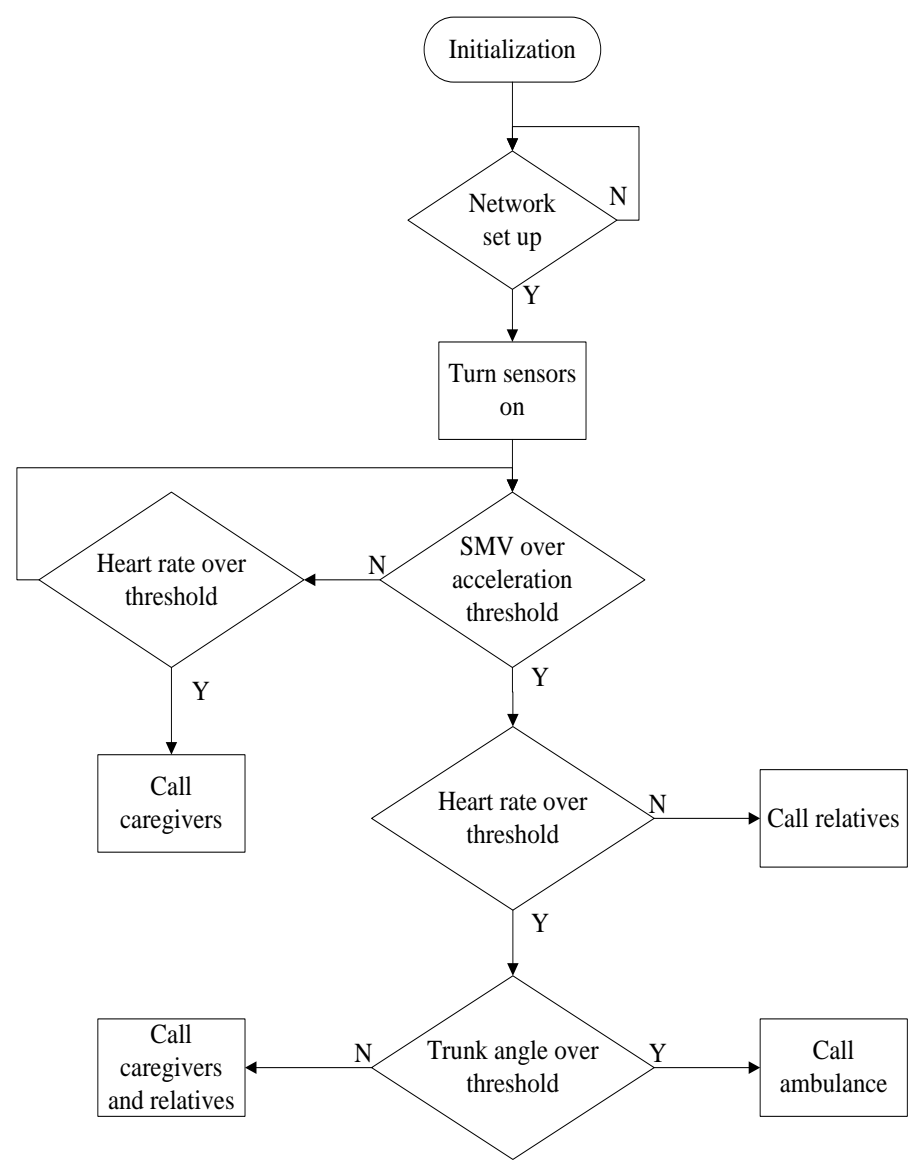

Fig. 6. Flowchart of using heart rate threshold minimizing the false positive rate of fall detection 


\section{Performance Evaluation}

\section{A. Laboratory Based Tests}

To evaluate the accuracy of proposed method, 30 healthy male and female participants were invited to take part in this research. Their ages range from 19 to 45 years, weights range from 48 to $80 \mathrm{~kg}$, and heights of 160 to $185 \mathrm{~cm}$. TABLE I lists 13 kinds of fall detection experiments including 6 falls and 7 activities representative of Active Daily Lives (ADL). In order to obtain meaningful data, participants are asked to perform every sub-experiment three times. The tests were accomplished by falling on thin mats in a configured laboratory. Their fall related data was transmitted to a laptop for further analysis.

As discussed in Section IV, SMV and trunk angle thresholds have been proposed by previous research. The threshold of heart rate changes needed to be carefully selected as the heart rate examination acts as the last classifier. Participants are asked to wear a pulse pressure sensor on their wrist and the integrated sensor board on their chest. After sensors are implemented carefully, participants were asked to do the tests set out in TABLE I. Two assistants stood next to the falling participant to make sure that there was no accident during the experiment procedure. After all tests, 900 meaningful results are chosen to calculate the upper limit of $95 \%$ confidence interval. Finally, heart rate change threshold can be set as $15 \%$. Fig. 7 depicts all the heart rate test results.

TABLE I

FALL EVENT AND ADL TESTS DESCRIPTIONS

\begin{tabular}{lll}
\hline \hline & No & \multicolumn{1}{c}{ EXPERIMENT } \\
\hline Fall & 1 & Backward fall, lying on ground \\
Fall & 2 & Backward fall, seating on ground \\
Fall & 3 & Backward fall, seating on chair \\
Fall & 4 & Forward fall, landing on knees \\
Fall & 5 & Forward fall, lying on ground \\
Fall & 6 & Seating in bed, falling to ground \\
ADL & 7 & Ascending stairs \\
ADL & 8 & Descending stairs \\
ADL & 9 & Running down the stairs \\
ADL & 10 & Walking and suddenly stop \\
ADL & 11 & Pick up an object from the floor \\
ADL & 12 & Fast stand up from a chair \\
ADL & 13 & Fast sit down to a chair \\
\hline
\end{tabular}

Fig. 7 illustrates the heart rate change before and after fall events. Participants under 30 years old have relatively small fluctuation when suffering a fall event. As to those over 30 years, the fluctuations are apparently high, which are $18 \%, 21 \%$, and $22 \%$ respectively. Therefore, if a fall event happened on an elderly person, the proposed heart rate threshold will normally be triggered. 


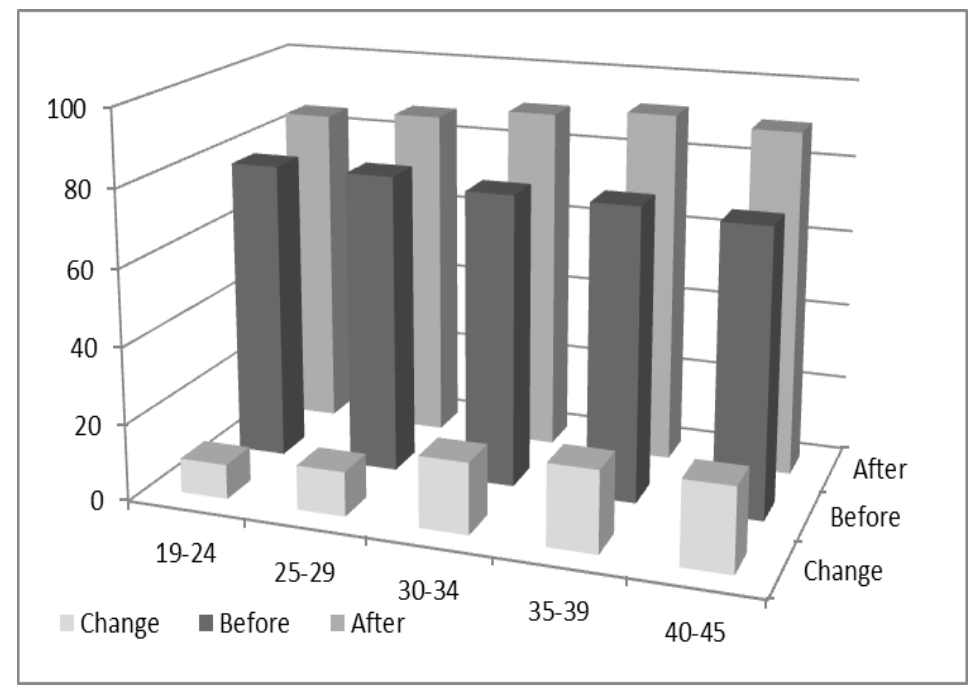

Fig. 7. Heart rate test results and changing ratio

As the system does not need any user feedback, the fall alarm can be sent within a few seconds. Fig. 8 shows the user interface in operation. As is shown in Fig. 8, the distinct vibration of the Acc curve illustrates a fall event may have occurred. In the meantime, the participant's heart rate changed from 62 to $74 \mathrm{bpm}$. Along with the backgrounder trunk angle calculation, a fall is alarmed as positive.

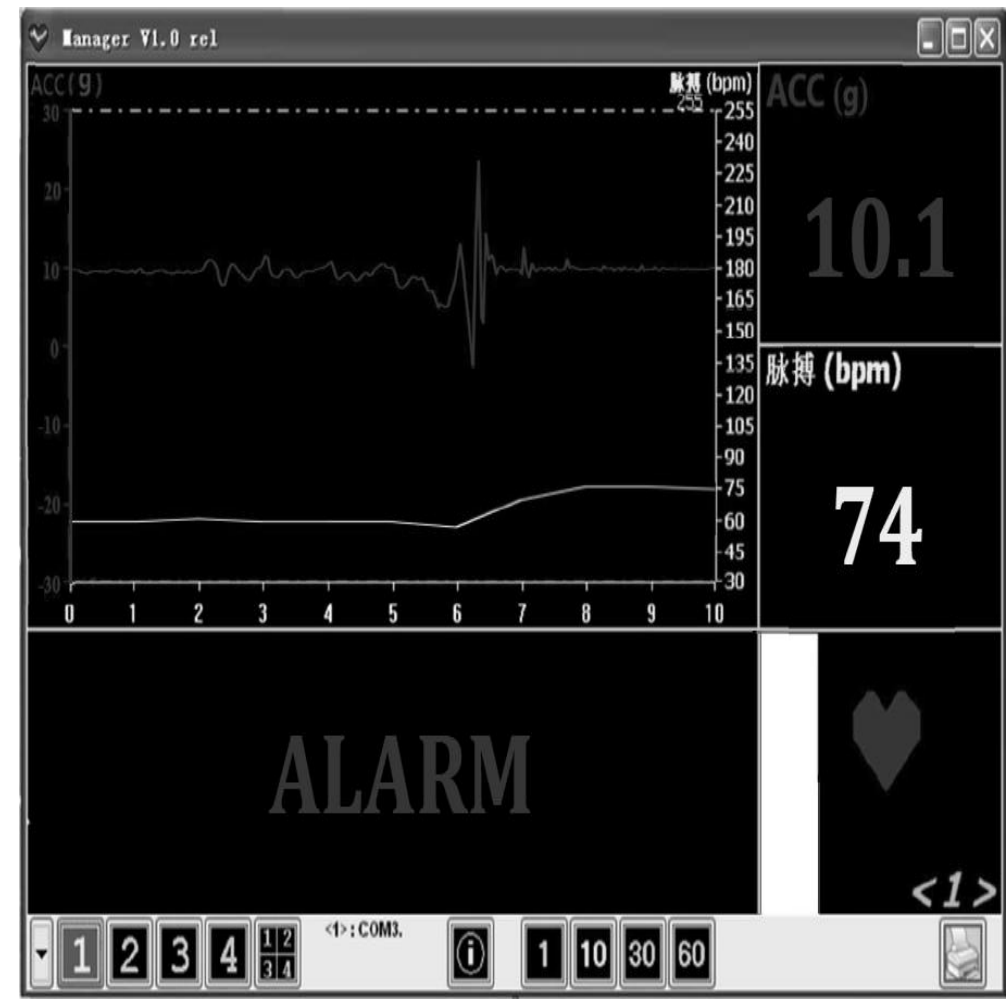

Fig. 8. GUI of Channel 1 in use. 
The definition of sensitivity and specificity are respectively given in equ. (3) and (4), where TP is the true positive and FN is false negative. Similarly, TN represents true negative and FP represents false positive.

Sensitivity $=\frac{T P}{T P+F N}$

Specificity $=\frac{T N}{T N+F P}$

As illustrated, sensitivity indicates likelihood of a fall event did occur, but had not been detected. On the contrary, represents the system triggered fall event alarm that actually had not happened.

Table II shows the detection results of the 6 prescribed fall events and Table III shows the detection results of the 7 prescribed ADL activities. The system accuracy was found to be $97.5 \%$. The sensitivity was found to be $96.8 \%$ and the specificity was found to be $98.1 \%$. A balance between sensitivity and specificity has thus been achieved

TABLE II

FALL EVENT TESTS RESUlts

\begin{tabular}{|c|c|c|c|}
\hline Fall & $\mathbf{T P}$ & FN & SENSITIVITY \\
\hline 1 & 90 & 0 & $100 \%$ \\
\hline 2 & 90 & 0 & $100 \%$ \\
\hline 3 & 89 & 1 & $98.9 \%$ \\
\hline 4 & 83 & 7 & $92.2 \%$ \\
\hline 5 & 90 & 0 & $100 \%$ \\
\hline 6 & 80 & 10 & $88.9 \%$ \\
\hline
\end{tabular}

TABLE III

ADL TESTS RESULTS

\begin{tabular}{|c|c|c|c|}
\hline Fall & TN & FP & SENSITIVITY \\
\hline 7 & 90 & 0 & $100 \%$ \\
\hline 8 & 90 & 0 & $100 \%$ \\
\hline 9 & 86 & 4 & $95.6 \%$ \\
\hline 10 & 90 & 0 & $100 \%$ \\
\hline 11 & 85 & 5 & $94.4 \%$ \\
\hline 12 & 87 & 3 & $96.7 \%$ \\
\hline 13 & 90 & 0 & $100 \%$ \\
\hline
\end{tabular}

The most inaccurate term in fall event tests tends to be due to falling out of bed. This may be caused by the fact that some participants subconsciously brake the fall with their arms causing low acceleration and therefore not reaching a trigger threshold. Also there are 8 false negatives reported in a forward fall, landing on knees and seating on a chair. After checking the raw data, this was because that trunk angle did not exceed the preset threshold. A lower trunk angle threshold may solve this issue. However, reducing the trunk angle threshold will cause the increase of false positive reports. This inconsistency always exists in threshold based systems. Thus, searching a balance between sensitivity and specificity is one very important issue under practical implementation. 
When using the system to do the ADL tests, 16 false positive reports existed, including running down the stairs, picking up an object from the floor, and standing up fast from a chair. Among the three events, the system missed 5 times in picking up an object from the floor. This was predominantly caused by fast head down movements that in turn caused marked changes in acceleration, trunk angle and heart rate. Four misses were found in running down stairs were caused by suddenly stopping at stair corners but as running is rare among elderly persons then these false positive reports were not considered further.

\section{B. Practical Tests}

The proposed system achieved a relatively high sensitivity and specificity in laboratory conditions. However, in order to validate the system in practical tests, the system was implemented with people aging from 5 to 70 years for two weeks, but as other researchers have found, there was no accidental fall that occurred when the system was deployed. However, during the 2-week monitoring a false positive alarm did not occur. A substantial amount of ADL data was collected which indicates the system to be stable and robust.

In future work, a new device with lower energy consumption and longer communication distance will be developed to make the system more suitable for a broad-range of healthcare applications.

\section{Conclusion}

In this paper, an enhanced fall detection system based on on-body smart sensors was proposed, implemented, and deployed that successfully detected accidental falls in a consumer home application. By using information from an accelerometer, smart sensor and cardiotachometer, the impacts of falls can successfully be distinguished from activities of daily lives reducing the false detection of falls. From the dataset of 30 participants, it is found that the proposed fall detection system achieved a high accuracy of $97.5 \%$, and the sensitivity and specificity are $96.8 \%$ and $98.1 \%$ respectfully. The proposed system is ready to be implemented in a consumer device.

\section{References}

[1] I. Akyildiz, W. Su, Y. Sankarasubramaniam, and E. Cayirci, "Wireless sensor networks: a survey," Journal of Computer Networks, vol. 38, no. 4, pp. 393-422, March 2002.

[2] J. Yick, B. Mukherjee, and D. Ghosal, "Wireless sensor network survey," Journal of Computer Networks, vol. 52, no. 12, pp. 2292-2330, Aug. 2008.

[3] K. Kinsella and D. R. Phillips, "Global aging: the challenge of success," Population Bulletin, vol. 60, 2005.

[4] Tabulation on the 2010 population census of the people's republic of China, China Statistics, May 2013, on-line.

[5] S. Demura, S. Shin, S. Takahashi, and S. Yamaji, "Relationships between gait properties on soft surfaces, physical function, and fall risk for the elderly," Advances in Aging Research, vol. 2, pp. 5764, May 2013.

[6] S. R. Lord and J. Dayhew, "Visual risk factors for falls in older people," Journal of American Geriatrics Society, vol. 49, no. 5, pp. 508-515, Dec. 2001.

[7] WHO, "The injury chart-book: a graphical overview of the global burden of injury," Geneva: WHO, pp. 43-50, 2012.

[8] M. Mubashir, L. Shao, and L. Seed, "A survey on fall detection: Principles and approaches," Neurocomputing, vol. 100, no. 16, pp. 144-152, Jan. 2013.

[9] Q. Zhang, L. Ren, and W. Shi, "HONEY a multimodality fall detection and telecare system," Telemedicine and e-Health, vol. 19, no. 5, pp. 415-429, Apr. 2013. 
[10] F. Bagalà, C. Becker, A. Cappello, L. Chiari, and K. Aminian, "Evaluation of accelerometer-based fall detection algorithm in real-world falls," PLOS ONE, vol. 7, no. 5, pp. 1-8, May 2012.

[11] S. Abbate, M. Avvenuti, F. Bonatesta, G. Cola, P. Corsini, and A.Vecchio, "A smartphone-based fall detection system," Pervasive and Mobile Computing, vol. 8, no. 6, pp. 883-899, Dec. 2012.

[12] S. Abbate, M. Avvenuti, G. Cola, P. Corsini, J.V. Light, and A.Vecchio, "Recognition of false alarms in fall detection systems," in Proc. 2011 IEEE Consumer Communications and Networking Conference, Las Vegas, USA, pp. 23-28, Jan. 2011.

[13] Y.W Bai, S.C. Wu, and C.L. Tsai, "Design and implementation of a fall monitor system by using a 3-axis accelerometer in a smart phone," IEEE Trans. Consumer Electron., vol. 58, no. 4, pp. 12691275, Nov. 2012.

[14] M. Yu, A. Rhuma, S. Naqvi, L. Wang, and J. Chambers, "A posture recognition-based fall detection system for monitoring an elderly person in a smart home environment," IEEE Trans. Infor. Tech. Biom., vol. 16, no. 6, pp. 1274-1286, Aug. 2012.

[15] C. Rougier, J. Meunier, A.S. Arnaud, and J. Rousseau, "Robust video surveillance for fall detection based on human shape deformation," IEEE Trans. Circ. Syst. for Video Tech., vol. 21, no. 5, pp. 611622, May 2011.

[16] M. Popescu, Y. Li, M. Skubic, M. Rantz, "An Acoustic Fall Detector System that Uses Sound Height Information to Reduce the False Alarm Rate", in Proc. 30th Int. IEEE Eng. in Medicine and Bio. Soc. Conference, pp. 4628-4631, Aug. 2008.

[17] J. Winkley, P. Jiang, and W. Jiang, "Verity: An Ambient Assisted Living Platform," IEEE Trans. Consumer Electron., vol. 58, no. 2, pp. 364-373, May 2012.

[18] H.R. Yan, H.W. Huo, Y.Z. Xu, and M. Gidlund, "Wireless sensor network based E-health system: implementation and experimental results," IEEE Trans. Consumer Electron., vol. 56, no. 4, pp. 2288-2295, Nov. 2010.

[19] D.M. Karantonis, M.R. Narayanan, M. Mathie, "Implementation of a real-time human movement classifier using a triaxial accelerometer for ambulatory monitoring," IEEE Trans. Infor. Tech. Biom., vol. 10, no. 1, pp. 156-167, Jan. 2006.

\section{BIOGRAPHIES}

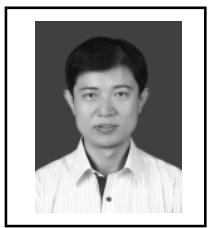

Jin Wang (M'11) received the B.S. and M.S. degree in the Electronical Engineering from Nanjing University of Posts and Telecommunications, China in 2002 and 2005, respectively. He received Ph.D. degree from Computer Engineering Department of Kyung Hee University Korea in 2010. Now, he is a professor in the Computer and Software Institute, Nanjing University of Information Science and Technology. He has published more than 100 journal and conference papers. His research interests mainly include routing protocol and algorithm design, performance evaluation and optimization for wireless ad hoc and sensor networks.

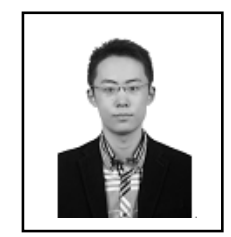

Zhongqi Zhang received the B.S. degree in the Electronic and Information Engineering from Nanjing University of Information Science and Technology, China in 2012. Now, he is working toward the M.S. degree in the Computer and Software Institute. His current research interests are in performance evaluation for wireless sensor networks, and healthcare with wireless body area networks. He is a student member of ACM and CCF.

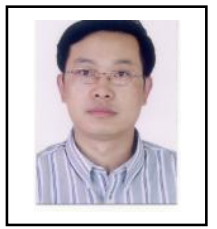

Bin Li received the B.S. degree in Computer Software from Fudan University, China in 1986, M.S. and Ph.D. degrees in Computer Application Technology from Najing University of Aeronautics \& Astronautics, China in 1993 and 2001 respectively. He is now a professor in Yangzhou University. He has published more than 100 journal and conference papers. His main research interests include artificial intelligence, multi-agent system and service oriented computing. 


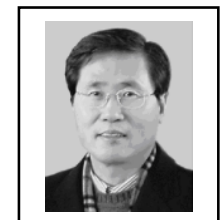

Sungyoung Lee received his B.S. from Korea University, Seoul, Korea. He got his M.S. and Ph.D. degrees in Computer Science from Illinois Institute of Technology (IIT), Chicago, USA in 1987 and 1991 respectively. He has been a professor in the department of Computer Engineering, Kyung Hee University, Korea since 1993. He is a founding director of the Ubiquitous Computing Laboratory, and has been affiliated with a director of Neo Medical ubiquitous-Life Care Information Technology Research Center, Kyung Hee University since 2006. Before joining Kyung Hee University, he was an assistant professor in the Department of Computer Science, Governors State University, Illinois, USA from 1992 to 1993 . His current research focuses on Ubiquitous Computing and Applications, Wireless Ad-hoc and Sensor Networks, Contextaware Middleware, Sensor Operating Systems, Real-Time Systems and Embedded Systems.

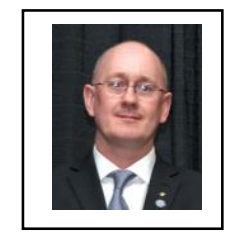

R. Simon Sherratt (M'97-SM'02-F'12) received the B.Eng. degree in Electronic Systems and Control Engineering from Sheffield City Polytechnic, UK in 1992, M.Sc. in Data Telecommunications in 1994 and Ph.D. in video signal processing in 1996 both from the University of Salford. In 1996, he has appointed as a Lecturer in Electronic Engineering at the University of Reading where he is currently a Professor of Consumer Electronics and Head of the Wireless and Computing research. He is also a Guest Professor at Nanjing University of Information Science and Technology, China. His research topic is on signal processing in consumer electronic devices concentrating on equalization and DSP architectures, specifically for Personal Area Networks, USB and Wireless-USB.

Eur Ing Professor Sherratt has served the IEEE Consumer Electronics Society as a Vice President (Conferences) (2008/9), AdCom member (2003-2008, 2010-) and Awards chair (2006/7). He is a member of the IEEE TRANSACTIONS ON CONSUMER ELECTRONICS Editorial Board (2004-) and is currently the Editor-in-Chief (2011-), the IEEE International Conference on Consumer Electronics general chair (2009) and the IEEE International Symposium on Consumer Electronics general chair (2004). He received the IEEE Chester Sall 1st place best Transactions on Consumer Electronics paper award for 2004 and the best paper in the IEEE International Symposium on Consumer Electronics in 2006. 